\title{
Ontology Driven Benchmarking on ITIL to achieve Six Sigma
}

\author{
Sanket Dash \\ Symbiosis Institute of Technology ,Pune
}

\begin{abstract}
Benchmarking helps in organizational gap analysis by comparing it with some predefined standard bench marks which instead allow process's system, capability to increase to the maximum level. Conglomerated with Ontology, it provides a source of precisely defined terms that can create a consensus of shared understanding concerning the domain of discourse pertaining to a certain domain of knowledge. With a contentious focus on the gap analysis accurate measures can be planned or maximum efficiency. In this context the Combined benefits of Information Technology Infrastructure Library (ITIL) for IT Service Management and Six Sigma defining a process improvement approach when adds to the bes usage of ontology driven benchmarking ensures a quality improvement strategy reducing operational costs to ensure bottom-line improvements. To add practicality to this operational issue of a service life cycle stage - Continual Service Improvement (CSI) is taken for consideration in order to measure and improve processes and services.
\end{abstract}

\section{General Terms}

Ontology, process improvements, service improvements

\section{Keywords}

\section{Benchmarking, Six sigma, ITIL, CTQ, CSI}

\section{INTRODUCTION}

Ontology is the art of being a simplified, abstract way of appreciating a piece of reality, indicating the reorganization of the existence of a set of objects and their interrelations for universal acceptance. In the context of Software process improvement with a view for continual incremental strategy effecting on issues found in an assessment and enhancing the efficiency of the processes by reducing the

risk and vulnerability; ontology plays a major role. By allowing Software process assessment to evaluate the performance of an implemented process system against a model, standard, or benchmark.

This adds a clear cut advantage to the model based approach to justify in the operational profile by describing the process improvement based on a model- or standard-based assessment results.[5]Rather in benchmark based strategy focus is given on the results based on assessments and gap analysis. The effective decision making and contradictions can be done for a software development organization's capability level which may end result in three relative levels: below, equal, or above the benchmark of each process

Ontologies molded together with process benchmarking in Software Engineering gives the best result by creating the standardizations (i.e. creation of information systems or intelligent agents);Offering a universally accepted consensus on the domain of discourse which creates an unambiguous approach for all the hidden assumptions related to a certain domain of knowledge [2].
A process model is a system that describes process organization, categorization, hierarchy, interrelationship , and tailor-ability." Wang [Wang 2000].Especially, the software process as defined by NASA Software Engineering Laboratory consists of a series of phases [Donzelli 2006] given as:

- Requirements Analysis: requirements changes, requirement increments

- Specification: specification changes, specification increments, specification correction reports.

High-level design: high-level design changes, high-level design increments, high-level design correction reports

- Low-level design: low-level design changes, low-level design increments, low-level design corrections reports

- Code: code changes, code increments, code correction reports.

- System-tested code: system-tested code changes, systemtested code increments, system-tested code corrections reports.

- Acceptance-tested code: acceptance-tested code changes, acceptance-tested code increments (final SW product).

This process model when collaborated with an ontology driven approach helps us in [21][23]:

1) Identification of the ontology development methodologies to cater to our established goal;

2) Identification of a life-cycle model best suited to the planned ontology development;

3) Identification of main inputs, outputs and activities to be performed in order to develop the aimed Ontology;

4) Identification of key activities in the ontology development process.

In order to maintain sufficient competence IT organizations need not to push all its software engineering processes to the highest level because it is neither necessary nor economic for every implementation[17]. Rather by the benchmark-based improvement approach [21][22], an optimized process improvement strategy identifies a sufficient and economic target process profile, which provides an organization with sufficient margins of competence in every process.[23]

In the process of aligning IT with business objectives organizations have turned to practices and quality methods that include the combined effect of IT Infrastructure Library (ITIL), and Six Sigma[3], and improve IT dependant business processes[2][7] by focusing largely on the customers. Six Sigma is a process improvement model that enables organizations to streamline processes by reducing the number of defects.[11] The sigma measure, $\sigma$, represents the standard 
deviation, which indicates the amount of variation or inconsistency in a process. The target for quality equates to six standard deviations from the mean — Six Sigma[12].

ITIL is a framework of best practices that can be used to assist organizations in developing their ITSM process-driven approaches[7]. It is a guide for establishing common processes, roles, and activities, with appropriate reference to each other and how the communication lines should exist between them. It is not a methodology. Organizations can use ITIL either in whole or in part, depending on their preferences. ITIL provides guidance on how to link existing processes and activities across IT departments in a structured context, and this linkage is key to quality service delivery.[3] The models provided in ITIL show the goals, general activities, inputs, and outputs of the various processes that can be tailored to the organization's needs. The intent is to establish a common language across functional areas. ITIL complies with requirements for ISO9001quality standards and is referenced by ISO9000 for IT standards.[4][5]

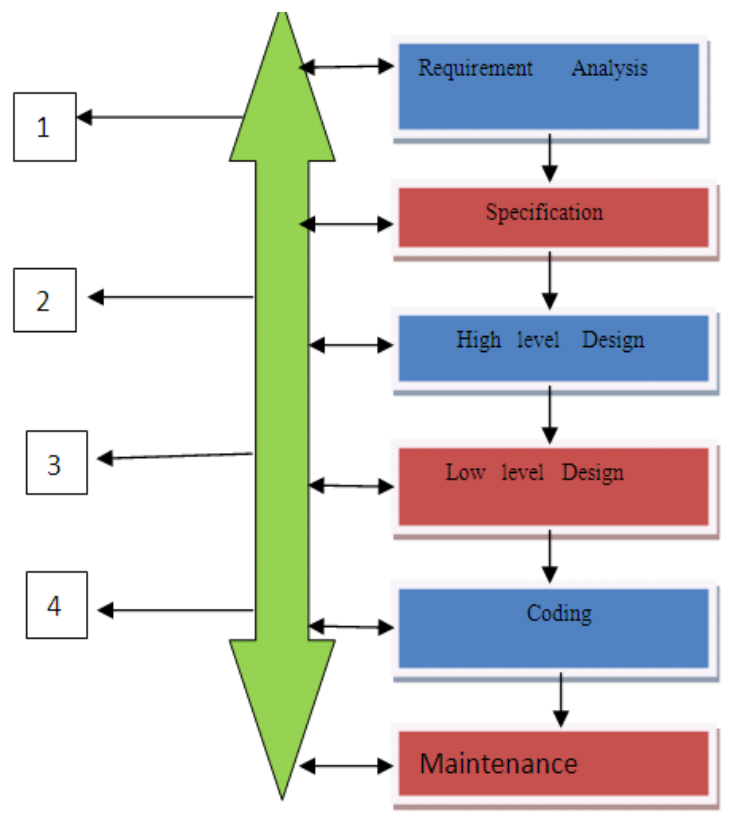

Figure-1:

Collaboration of ontology on a process model to carry out effective bench marking $(1,2,3,4$ points are elaborated below)

\section{2 .RELATED WORK}

The software process approach conglomerates the concepts of systematic, organizational and managerial infrastructures for software engineering. Sometimes it necessities to expand the scope for achieve high reliability, profit and customer satisfaction. Process Based Software Engineering was originally introduced as a software management method [Humphrey and Sweet 1987; Humphrey 1988; Gilb 1988], a quality assurance approach [Evans and Marciniak 1987; ISO 9001 1989], or as a set of software development techniques [Curtis et al. 1987; Fayad 1997].

The adoption and so as the development of CMM and of ISO 9000 , software engineering process diversify towards foundations, modeling, establishment, assessment, improvement, benchmarking, and standardization up to a greater extent. Process may be classified as a set of linked activities that take an input and transform it to create an output.

The software engineering process as a system is no different; it takes a software requirement as its input, while the software product is its output. But the mystery is that all the inputs and outputs are intangible and hence highly volatile if a proper cause and effect is not estimated. That actuates the need of statistically relating the total scenario on some well defined and wel practiced management frame work like ITIL.[3] ITIL defines a framework for IT Service Management and contains of a set of guidelines that specify what an IT organization should do based on industry best practices,fig-1. On the contrary Six Sigma defines a process improvement approach that is based on statistical measurement, drives quality improvement, and reduces operational costs.[2]

In total, ITIL defines the "what" of service management and Six Sigma defines the "how" of quality improvement. Together, they make a great combination for improving the quality of IT service delivery and support as well evaluated at GE [7] in accordance to IEEE standards [6] due to its backward compatibility with ISO and other well defined process models. Nowadays, many software development organizations want to improve their software quality by adoption of a process improvement program, but in reality, their positive effects are not very evident [16]. The main reasons as follows[8][9]:

1) Absence of a unified software process improvement framework on the improvement aspect.

2) Absence of the common knowledge framework for the usage and adaptability to clearly explain the implemented process.

3) Trivial and lengthy software process improvement methods which makes hard to tolerate for small or medium-sized organizations [9].

4) Lack of effective support environment for proper execution and implementation.

\section{SIX SIGMA}

The American Society for Quality (ASQ) defines quality as "a subjective term for which each person has his or her own definition (American Society 2002). In technical usage quality has two meanings: (i) the ability to satisfy stated or implied needs and (ii) a product or service free of deficiencies . The core Japanese principle "doing things right all the time" [10] may be applicable to a production process where one can limit the variability mechanically, but incase of a service paradigm the quality is just customer oriented and it involves measure of customer satisfaction. 


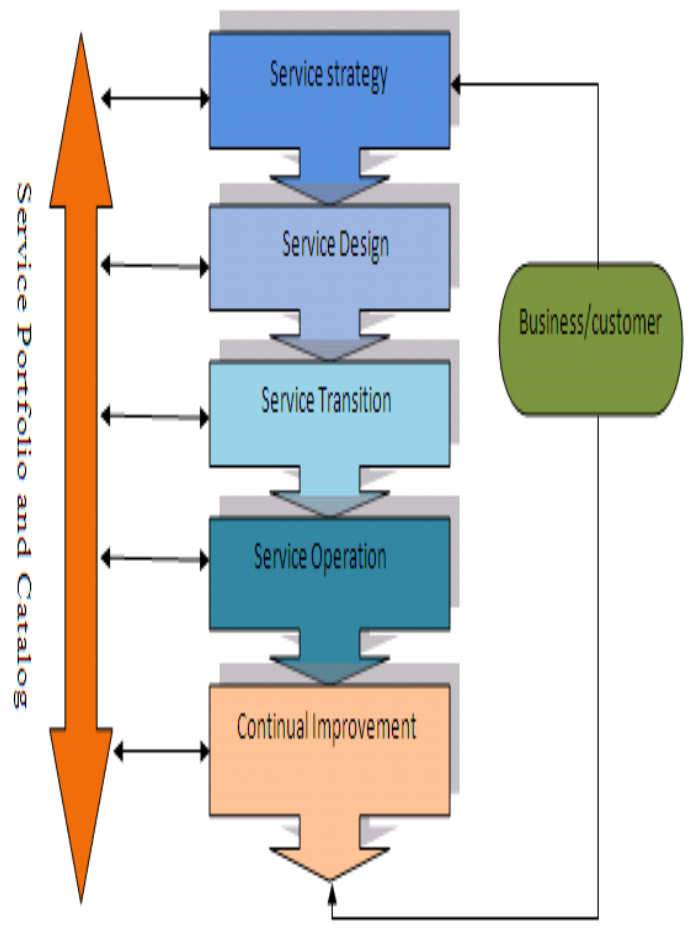

Fig-2:

\section{ITIL SERVICE DELIVERY PARADIGM}

Sometimes 99\%[1][10] in quality is not good enough consider .In case of IT, where everything is so fragile and intangible the quality is of course is a point of concern...Moreover looking at the Reliability aspects of the IT processes and Products we need to go through a statistically defined process for aligning technology and process to achieve higher quality.[11]

Six Sigma is a quality paradigm that works on aligning the internal procedures for reducing processes variability with customers' expectations. This helps organizations derives from the significant financial results provided [11]. For the software developer companies that apply maturity models, it has shown to be the ideal complement in quantitative management and in continuous process improvement [12]. Again, having a statistical base it can be better mapped to adaptive process improvement methodology to measure the process variance and so increase the process capability.

Six Sigma is accounted on two basic approaches, an administrative and a technical one. The first is directed to the choice of the problem to be solved or to the process to be improved, the goals to be attained, the aims to be followed, the projects to be executed and the people with the necessary abilities to successfully execute the improvement projects [14]. The technical approach focuses on processes improvement, with an increase in the average performance level and with a reduction in variability [13]. The maturity of a manufacturing process can be described by a sigma rating indicating its yield, or the percentage of defect-free products it creates. A six-sigma process is one in which 99.99966\%[1][10] of the products manufactured are statistically expected to be free of defects (3.4 defects per million).[13][14] Motorola set a goal of "six sigma" for all of its manufacturing operations, and this goal became a byword for the management and engineering practices used to achieve it. In case of service offerings it creates a special infrastructure of people within the organization ("Black Belts", "Green Belts", etc.) who are experts in these methods. Each Six Sigma project carried out within an organization follows a defined sequence of steps for process mapping ,process evaluation and that not only has quantified financial targets (cost reduction or profit increase) but also has other benefits such as reduction in errors or [mistake proofing] resulting in better controls on the business processes. Motorola developed the first Six Sigma implementation method aiming to solve a series of problems. The method was developed by Dr. Mikel Harry and consisted in four stages: Measure (assessment of data collection about the present process situation), Analysis (understanding the causes of the present performance of a process), Improvement (elaboration of improvement alternatives in the process performance) and Control (procedures to keep the improvements obtained and to make them long-lasting) [16]. GE, unlike Motorola, did not count on a perfect knowledge of the problems and, therefore, had to initially map what the quality problems were to solve them in the sequence. Moreover, it intended to use Six Sigma for the new problems, and thus the method should also contemplate problem identification. Taking advantage of the experience acquired at Motorola, Dr. Harry elaborated the DMAIC (Define, Measure, Analyze, Improve and control) method,fig2 , including in the original method an initial stage for defining the problem to be solved [15]. The software process management consists of two major aspects in contrast to six sigma[20]. On the first case, design of six sigma is used in software requirement engineering process. a model based on the IDOV (Identify, Design, Optimize, Verify)process is built for the requirement analysis which is in order to prevent defects from the very beginning of software life cycle. On the other hand, the DMAIC process is applied to optimize the whole software engineering process.

\section{SW-DMAIC:}

The SW-DMAIC [19] broadly classifies three groups of processes, corresponding to the Six Sigma implementation in organizations:

(a) Introduction and motivation when the company has the first contact with Six Sigma method and decides to adopt it;

(b) Generative feedbacks, when the company starts a new Six Sigma project and it is necessary to align all the developing team;

(c) Implementation of each of the Six Sigma projects, when the company starts a new project for achieving the Six sigma factor.

The DMAIC philosophy is carried down by these steps in a elaborative way as

- Definition: identification of problems and situations to improve and finding way for critical to quality (CTQ);

- Measurement: selection of critical characteristics to quality (CTQ) through necessary measures in the process; 


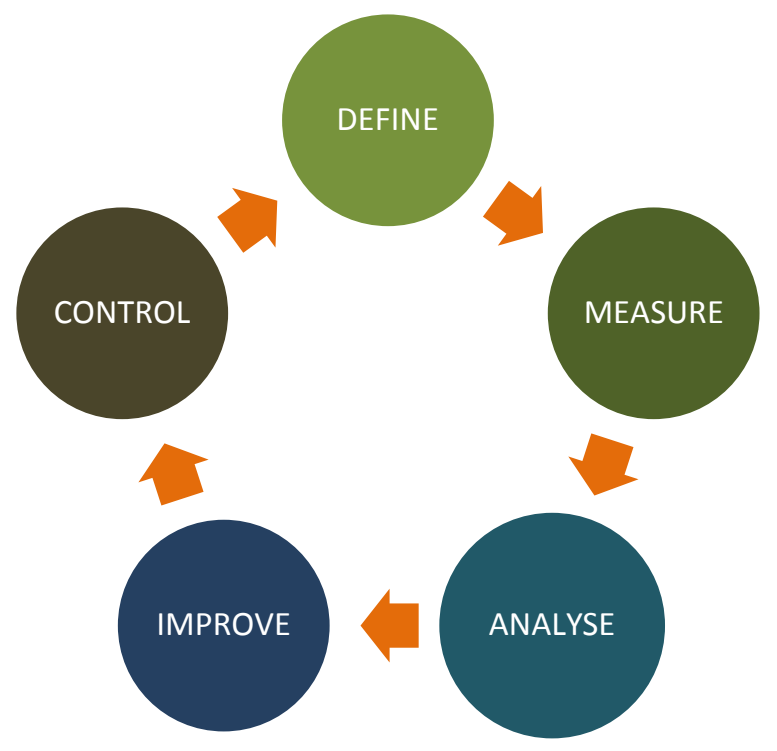

Fig.-3:The DMAIC lifecycle

- Analysis: identification of the root-causes that are responsible for the identified problems;

- Improvement: specification of process characteristics to be improved;

- Control: documentation and follow-up of the new process conditions.

Requirements engineering process is a very important phase of software engineering and also highly essential for achieving Six Sigma in the IT end product. . The IDOV philosophy is supposed to be the best way to map these process statistically to estimate the variations in a IT process improvement. It is generally carried down in this way:

- IDENTIFY : Identify customer Collect VOC

- DEFINE : Confirm CTQ

- OPTIMISE : Evaluate risk

- VERIFY : verify process, finish requirement document.

\section{RESTRICTIONS FOR SIX SIGMA FOR SOFTWARE DEVELOPMENT PROCESS}

The major restrictions faced with this six sigma topology in $\mathrm{s} / \mathrm{w}$ development paradigm [19] are: (a) diversity of requirements and usage from different clients for the same functionality of software; (b) The intangible IT business process; (c) non-normal statistical distributions of defects and of the use of the resources employed; (d)Reliability; (e) uncontrolled variations ie several main causes for the same variability, such as: alteration in technology, degradation in processing due to the equipment, communication networks, data volume, etc; (f) lack of company maturity, mainly in small organizations, understanding as superfluous the investments in process improvement; (g) lack of staff practice for applying statistical techniques; (h) extreme resistance to the alterations in development processes(i)The people factor [17] [18].

\section{ITIL FRAME WORK FOR QUALITY}

ITIL stands for the IT Infrastructure Library. It documents best practices for IT service management and is used by many organizations around the world, though originated as government initiatives in the UK and Holland. ITIL consists of a series of books giving guidance on the provision of quality IT services, and on the accommodation and environmental facilities needed to support information technology. The books that make up the ITIL library are delivered under the overall guidance of the UK Office of Government Commerce (OGC)) although ITSMF members play such a significant role in the development of these manuals, that this should be seen as a collective effort fig-3. It contains an integrated set of well-documented modules that include:

- Change Management

- Configuration Management

- Problem Management

- Helpdesk

- Service Level Management

- Security Management

- Capacity Management

- Availability Management

- Cost Management

- Network Services Management

- Software Control \& Distribution

- Outsourcing

According to best practices by companies like GE, MOTOROLA and well documented effort from PROXIMA Technology organizations adopting for ITIL best practices, Six Sigma brings three essential benefits:

1. It provides process for project implementation and management.

2. It helps IT organizations focus service improvement on areas that will have the maximum payback

3. It provides a set of techniques that can be used to establish a rating of quality, isolate problems and then make changes to improve the process.

\section{FRAMEWORK FOR CONTROL THROUGH DMAIC}

DEFINE: Catalogue Services, Draft, Negotiate, Review UCs and OLAs Agree SLAs.

Supporting Techniques: project charter, stakeholder analysis, SIPOC (suppliers, inputs, outputs and customers), rolled throughput yield, affinity diagram, Kano model, critical to quality tree.

MEASURE: Failure Mode and Effect Analysis (FMEA) corresponding to a service in ITIL Service Delivery.

Supporting Techniques: data collection, control charts, frequency plots, gage R\&R, Pareto charts, prioritization matrix, FMEA, process capability, process sigma, sampling, stratification, time series.

ANALYZE: baseline performance of the process is evaluated (CTQ processes) with this data being gathered by the service level 


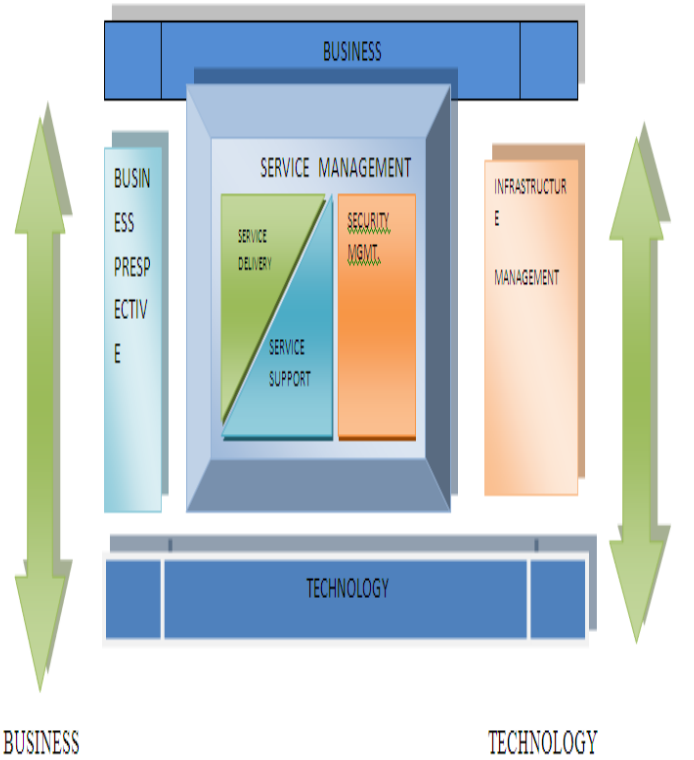

Fig-4 ITIL Publication Framework (source: OCG) management tool for better monitor report and review. Supporting Techniques: affinity diagrams, brainstorming, cause-and-effect diagrams, control charts, data collection, design or experiments, flow diagram, frequency plots, hypotheses tests, Pareto chart, regression analysis, response surface method, sampling, scatter plots, stratified frequency plots.

IMPROVE: Review Service Level Agreements Operation Level Agreements and Use Cases.

Supporting Techniques: Brainstorming, consensus, creativity techniques, data collection, design of experiments, flow diagrams, FMEA, hypothesis test, planning tools, stakeholder analysis.

CONTROL: Review Service Level Management Process Supporting Techniques: Control charts, data collection, flow diagrams, charts to compare before and after such as frequency plots, Pareto charts, quality control process chart, and standardization. The total ITIL and Six sigma process can be better summed up as given in Fig-4.

ITIL

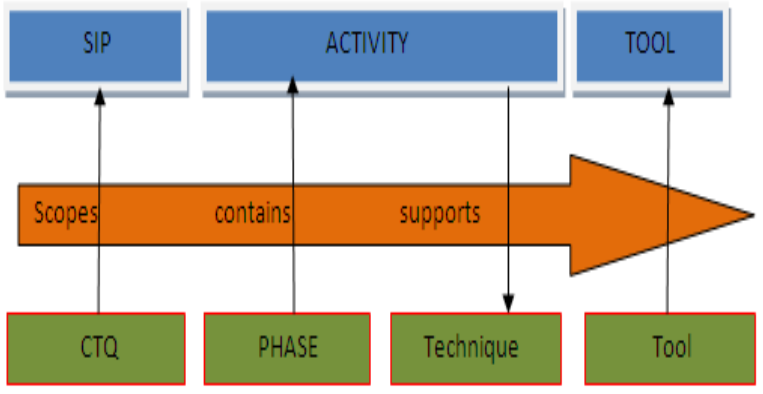

SIX SIS:II

Fig-4: ITIL and SIX Sigma Relationship

\section{BENCHMARK IMPROVEMENT \\ BASED \\ PROCESS}

Software process improvement methodologies are classified into two types: model-based and benchmark-based.[8] Model based software improvement strategy stress on the philosophy" The higher the better." It helps in improving a process system from a given level in a defined scale to a higher level. The benchmark based strategy identifies the gaps between development organizations process system and compares with a specified bench marks. In this approach process system's capability is improved towards an optimum profile, rather than the maximum Capability level, because it is neither necessary nor economic [9]. The paradigm shift in the philosophy revolves around 'Balance and Optimum'.This yields a highly reliable and qualitative process. One certain thing derived from Darwin's principle:"quality begets quality". If the process is itself standardize and of high quality then the end results in most of the cases will be of high quality. In case of some small variations we can control those parameters to achieve a high quality factor. The benchmarkbased process improvement creates procedure is a continuous spiral like, which can be carried out in 10 major steps as below [8]:

\section{1) Initial Assessment}

Projects and organizations have their own specific characteristic, so an initial assessment is necessary to evaluate the baseline efficiency and effectiveness of the Software Process Improvement Strategies

\section{2) Setting Scope and Goal Level}

It adopts a set of relative and dynamic target capability levels and the benchmarks and does not specify an absolute higher capability level as the target capability level. So that the process can balance and optimize to the maximum extent

3) Gap Analysis: The current level and the goal level benchmarks curves are analyzed to find out the gaps, so that, the improvement opportunities will be found out.

\section{4) Retrieval of candidate process models}

Retrieval a set of candidate process model fragments from repository with the reusable objects accumulating makes the development be more efficient.

\section{5) Building a process model}

An initial process model will be built to execute under the support environment.

\section{6) Process enactment with domain knowledge}

Domain knowledge is used to fill the gap between incomplete process model and the real requirements through expert advice, brain storming and gap analysis.

\section{7) Monitor}

Through monitoring on statistical variations and financial assessment is required in order to ensure tasks progress as expected and implementation is correct.

\section{8) Validate}

Validation is required to confirm whether the planned improvement goals and target capability levels have been achieved. Measurements of process effectiveness may be used to confirm achievement of process effectiveness targets. The variations from the majot goals are also calculated.

\section{9) Update}

By the process enactment more knowledge and understanding will be gained and these process models and the relative knowledge should be versioned, updated and stored in a repository for the next reuse.

\section{0) Next round improvement}

Through the continual learning and improvement process, feedback in the small cycle is conducted for monitoring, supporting, and resolving the problems encountered during the 
execution of process which end results as accumulated experiences and models, prepared for the next reuse.The total system can be better elaborated and detailed as given in Fig-5

\section{CONCLUSION}

Software processes are only successful if they are built up with a given consideration on the three factors, i.e. people, process and the product. Majority of the software development organizations have been slow to implement audit process improvement and recommendations.

ITIL and Six Sigma appear to be mutually exclusive but recent implementation in GE and Motorola illustrates its combined power as a frame work to increase the quality and the reliability. However, as this paper discusses how the ontology driven bench marking can further enhance the productivity of the total paradigm in terms of process improvement. Moreover, good practices, improvement strategies on project validates good enhancement in productivity. Further, these approaches are highly complementary and can be used in combination effectively to continually improve business processes and reduce costs by helping to minimize potential downtime and the adverse effects of system, network, and application failures .Enhance decision-making ability by facilitating access to information throughout the organization. But practically elongated and enterprise wide use of outputs from an integrated framework of processes and tools are not that helpful if the managerial decision making is not adhered to quality. Moreover effective statistical analysis should be taken into place in order to measure the actual productivity and efficiency of the point of discussion. The working model has to be set for its implementations in small and medium so as the large software installations to exactly evaluate the enhanced efficiency.

Approaches like though consider carefully about the process efficiency, still continuous research should be done on the project management aspects so as to bring the theory into practicality.

IT service levels can be improved by creating operational efficiencies and enabling a linked IT Service Management process loop for defining, measuring, analyzing, improving, and controlling service performance, but still there is no statistical measure to evaluate the impact on the total improvement. The point of discussion revolves around some statistical input the selection of these inputs and the actual measurement of the process need to be considered. Moreover, more brain storming is required to parameterize the decision statistically so that the decision maker can

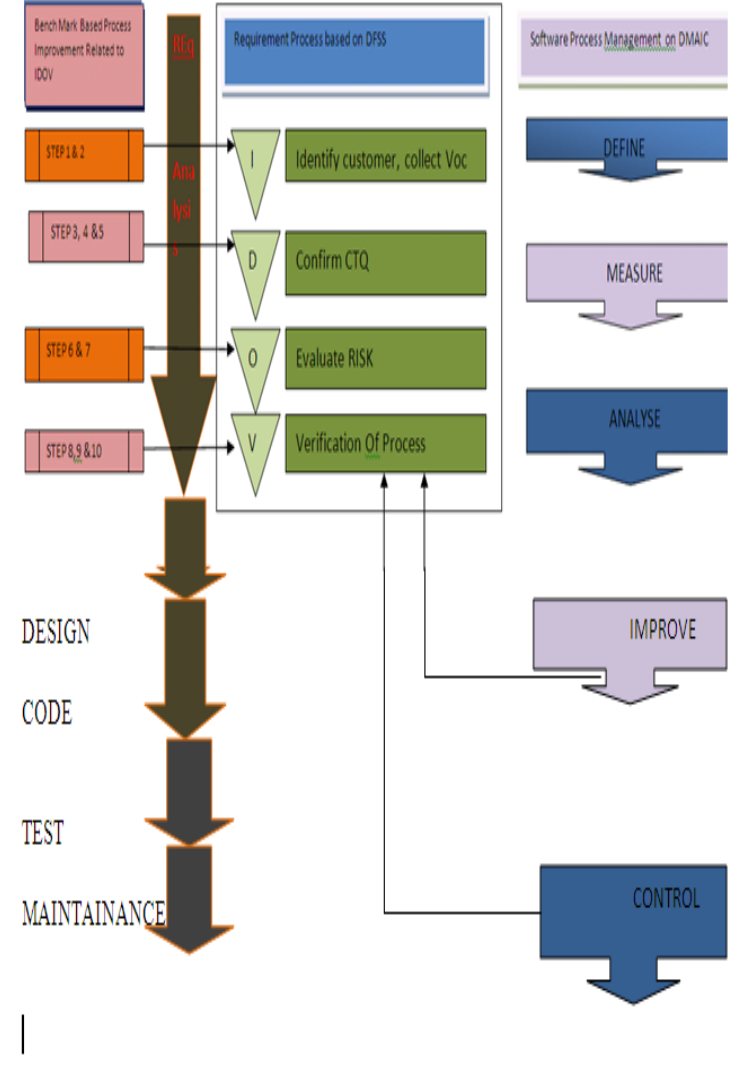

Fig -5:

\section{ONTOLOGY DRIVEN BENCHMARK BASED SOFTWARE PROCESS MANAGEMENT WITH SIX SIGMA}

reach up to the six sigma level with all the operational process not through tradeoff but through concrete steps.

\section{REFERENCES}

[1] Study and analysis of control phase role for increasing the Success of six sigma projects Yousef Gholipour Kanani, 1-4244-0148-8/06IEEE

[2] Six Sigma for IT Service Management, SixSigmaZone.com

[3] An Overview of the Adoption of IT Governance Models and Software Process Quality Instruments at Brazil Preliminary Results of a Survey- Rodrigo Santos de Espindola, Edimara Mezzomo Luciano, Jorge Luis Nicolas Audy, Proceedings of the 42nd Hawaii International Conference on System Sciences - 2009

[4] Software Process Standardization for Distributed Working Groups Carmen Maidantchik, Ana Regina C. Rocha and Geraldo Xex'eo COPPE - Universidade Federal do Rio de Janeiro

[5] SOFTWARE PROCESS THEMES AND ISSUES ,Mark Dowson, Marlstone Software Technology Inc 0-81863600-9/93 1993 IEEE

[6] SOFTWARE DEVELOPMENT PROCESS STANDARDS: Challenges for Process Assurance R. Craig Smith Motorola, Inc.1082-3670/97 1997 IEEE 
[7] White paper: Combining ITIL and Six Sigma to improve Information Technology Service Management at General Electric

[8] A Methodology and its Support Environment for Benchmark-based Adaptable Software Process Improvement , Wu Minghui, 'k'ing Jing, Yu Chunyan , 2004 IEEE international Conference on Systems, Man and Cybernetics

[9] Y. Wang, G. King, "A New Approach to BenchmarkBased Process Improvement", Proceedings of European Software Process Improvement 2000, Copenhagen, pp.140-149, Nov. 2000.

[10] Waxer (2006), Six Sigma costs and savings Aug2009 .http://www.isixsigma.com

[11] Hunter, D.; B. Schmidt, Six sigma: benefits and approaches, Chemical Week, 161, 37, 35-36, 1999.

[12] Murugappan, M.; Keeni, G., Quality improvement: the Six Sigma way, 2000, Proc. Of Asia Pacific Conference

Quality Software, IEEE CS Press, Los Alamitos, 248257.

[13] Marash, S. A., Six Sigma: business results thoughinnovation, 2000, Proc of the 54th Annual Quality Congress of the American Society for Quality, Indianapolis 627-630.

[14] Harry, M., Schroeder, H., Six Sigma: a breakthrough strategy for profitability, Quality Progress. 31, 51998.

[16]Blakeslee Jr., J. A., 1999, Achieving quantum leaps in quality and competitiveness: implementing the Six Sigma solution in your company, Proc. Of THE 53TH
ANNUAL QUALITY CONGRESS OF THE AMERICAN SOCIETY FOR QUALITY, Anaheim: Califórnia, 486-496.

[17] Biehl, R. E., Six Sigma for software, Quality time,

IEEE Software, 21, 2, 68-70, 2004.

[18] SW-DMAIC: A SIX SIGMA NEW GENERATION FOR IMPROVING SOFTWARE DEVELOPMENT PROCESSES, A.C. Tonini, F.J.B.Laurindo, M.M.Spínola , 19th International Conference on Production Research

[19] Research on the Application of Six Sigma in Software Process Improvement, ZHAO Xiaosong, HE Zhen, GUI Fangfang, ZHANG Shenqing, International Conference on Intelligent Information Hiding and Multimedia Signal Processing.

[20] T.R. Gruber. Towards Principles for the Design of Ontologies Used for Knowledge Sharing. In Roberto Poli Nicola Guarino, editor, International Workshop on Formal Ontology, Padova, Italy, 1993. Technical report KSL-93-04, Knowledge Systems Laboratory, Stanford University.

[21] Gruninger, M., Lee, Jintae., Ontology Design and Applications, Communications of the ACM, February 2002, 45 (2), 1-2, 2002.

[22 ] C. Wille, A. Abran, J-M Desharnais, R. Dumke, The Quality concepts and sub concepts in SWEBOK: An ontology challenge, in International Workshop on Software Measurement (IWSM) , Montreal , 2003 , pp. 18 